\section{IC-P2-128 TWELVE-MONTH CEREBRAL METABOLIC DECLINES IN PROBABLE ALZHEIMER'S DISEASE AND AMNESTIC MILD COGNITIVE IMPAIRMENT: PRELIMINARY FINDINGS FROM THE ALZHEIMER'S DISEASE NEUROIMAGING INITIATIVE}

Eric M. Reiman ${ }^{1,2}$, Kewei $\mathrm{Chen}^{3,4}$, Napatkamon Ayutyanont ${ }^{3,4}$, Wendy Lee ${ }^{3,4}$, Dan Bandy ${ }^{3,4}$, Cole Reschke ${ }^{3,4}$, Gene E. Alexander ${ }^{2,4}$, Michael W. Weiner ${ }^{5}$, Robert A. Koeppe ${ }^{6}$, Norman L. Foster ${ }^{7}$, William J. Jagust ${ }^{8},{ }^{1}$ Banner Alzheimer's Institute, Arizona Alzheimer's Consortium, Translational Genomics Research Institute, Phoenix, AZ, USA; ${ }^{2}$ University of Arizona, Tucson, AZ, USA; ${ }^{3}$ Banner Alzheimer's Institute, Phoenix, AZ, USA; ${ }^{4}$ Arizona Alzheimer's Consortium, Phoenix, AZ, USA, ${ }^{5}$ University of California, San Francisco, CA, USA; ${ }^{6}$ University of Michigan, Ann Arbor, MI, USA; ${ }^{7}$ University of Utah, Salt Lake City, UT, USA; ${ }^{8}$ University of California, Berkeley, CA, USA. Contacte-mail: Eric.Reiman@bannerhealth.com

Background: In a small positron emission tomography (PET) study, we previously characterized twelve-month cerebral metabolic rate for glucose (CMRgl) declines in moderate probable Alzheimer's disease (pAD) patients and estimated the number of patients needed to detect diseasemodifying treatment effects in a single-center randomized clinical trial (RCT, Alexander et al, 2002). The objectives of this study are to characterize twelve-month CMRgl declines in mild pAD and amnestic mild cognitive impairment (aMCI) patients from the multi-center Alzheimer's Disease Neuroimaging Initiative (ADNI) and estimate the number of mild pAD patients and MCI patients needed to evaluate a putative diseaseslowing treatment in twelve-month multi-center RCTs. Methods: SPM5 was used to characterize twelve-month declines in 17 mild pAD patients (baseline age $71 \pm 12$, MMSE $24 \pm 2$ ) and 46 aMCI patients (baseline age $66 \pm 13$, MMSE 27 \pm 2 ). Maximal CMRgl declines were used to estimate the number of mild $\mathrm{pAD}$ and aMCI patients needed per group to detect disease-modifying treatment effects in six- or twelve-month multi-center RCTs with $80 \%$ power and $\mathrm{P} \leq 0.001$ uncorrected for multiple comparisons. Results: The pAD patients had twelve-month CMRgl declines in posterior cingulate, precuneus, parietal, temporal and frontal regions and the aMCI patients had twelve-month CMRgl declines in the posterior cingulate, precuneus, parietal and temporal regions. To detect a $20 \%$ treatment effect on posterior cingulate $\mathrm{CMRgl}$ declines, we estimate the need for 153 mild pAD patients per group and $728 \mathrm{aMCI}$ patients per group in twelve-month RCTs. Conclusions: This study provides preliminary information about twelve-month CMRgl declines in mild pAD and aMCI patients and the number of patients needed to detect disease-modifying treatment effects in a twelve-month multi-center RCT. Future analyses will extend our findings to the entire ADNI cohort, provide power estimates for 6, 12, 18 and 24-month multi-center RCTs using specified search regions versus ROIs, and compare these power estimates to those using clinical ratings, other imaging modalities and other image-analysis techniques.

\section{IC-P2-129 VOLUME LOSS AND PARAHIPPOCAMPAL} WHITE MATTER INTEGRITY IN AMNESTIC-MCI

Emily J. Rogalski ${ }^{1}$, Chris M. Murphy ${ }^{1}$, Leyla deToledo-Morrell ${ }^{1}$, Raj C. Shah ${ }^{2}$, Mehul A. Trivedi ${ }^{1}$, Chengsheng Wang ${ }^{1}$, Glen T. Stebbins ${ }^{1},{ }^{1}$ Rush University Medical Center Department of Neurological Sciences, Chicago, IL, USA; ${ }^{2}$ Rush University Medical Center Departments of Family Medicine and the Rush Alzheimer's Disease Center, Chicago, IL, USA. Contact e-mail: erogalski@gmail.com

Background: Volume loss in mesial temporal lobe structures (e.g. hippocampus and entorhinal cortex) as well as the parahippocampal white matter (PWM) region has been demonstrated in individuals with mild cognitive impairment (MCI) and Alzheimer's disease (AD) and is thought to contribute to memory decline in elderly individuals. It is unclear whether the volume loss in the PWM region of individuals with MCI is accompanied by microstructural alterations in remaining white matter. Diffusion tensor imaging (DTI) makes it possible to examine the microstructure of white matter in vivo and is especially indicative for diseases causing neuronal or axonal damage. The technique is based on sensitizing the magnetic resonance (MR) signal to movement of water on the order of several microns (diffusion weighted MRI) and measuring the direction and magnitude of water diffusion in six noncollinear gradients. Methods: The present study explored the integrity of the PWM region that includes the perforant path in 18 amnestic MCI subjects compared to 14 elderly controls (NCI) using two different high resolution DTI protocols: 1) a whole-brain scan with 38-slices (3mm thick), and 2) a 13-slice scan (2mm thick) of the temporal lobe that includes the critical PWM region. The PWM region of interest (ROI) was traced bilaterally for each subject using ANALYZE software on high resolution T1-weighted 3D-SPGR scans. All images were acquired on a $1.5 \mathrm{~T}$ scanner. Structural images and ROI's were coregistered to the DTI scans using SPM2 and DTI values were extracted using laboratory-based software. Results: In agreement with previous findings from our laboratory, results showed significantly smaller PWM volume in MCI compared to NCI subjects $(p<0.001)$. However, none of the DTI measures (Lattice Index, Mean Diffusivity, Coherence Index, Fractional Anisotropy, primary and radial eigenvalues) of the PWM showed significant group differences in either DTI protocol. Conclusions: These results suggest that in MCI volume loss in the PWM is a consequence of a decrease in the number of fibers rather than a disruption of their microstructure. It is possible that microstructural alterations in remaining PWM fibers may come with time and be a critical factor in the conversion from $\mathrm{MCI}$ to $\mathrm{AD}$.

\section{IC-P2-130 PROFILES OF LONGITUDINAL BRAIN ATROPHY IN SEMANTIC DEMENTIA}

Jonathan D. Rohrer, Elizabeth McNaught, Jo Foster, Shona Clegg, Josephine Barnes, Rohani Omar, Martin Rossor, Jason Warren, Nick Fox, Institute of Neurology, London, United Kingdom. Contact e-mail: rohrer@dementia.ion.ucl.ac.uk

Background: Semantic dementia (SD) is a sporadic neurodegenerative disorder characterized clinically by progressive erosion of semantic processing in verbal and nonverbal domains and pathologically by the presence of ubiquitinpositive, TDP-43 positive neuronal inclusions. Brain imaging typically shows asymmetrical antero-inferior temporal lobe atrophy more severe on the left. Within the spectrum of the frontotemporal lobar degenerations (FTLD), the uniform and specific clinico-pathological signature of SD makes it the leading candidate FTLD subtype for trials of disease-modifying therapy. The aims of this study were to characterise in detail the pattern of global and regional longitudinal brain atrophy in SD and to identify imaging biomarkers of SD that could underpin therapeutic trials. Methods: In a cohort of 21 patients with SD (including 8 with pathological confirmation) we performed whole brain and region of interest analyses on volumetric brain MRI scans at two time-points. Results: Mean whole brain atrophy rate measured using the boundary shift integral (BSI) was $2.5 \%$ per year $(0.4 \%$ per year in controls) with ventricular enlargement at $6.2 \mathrm{ml}$ per year $(0.6 \mathrm{ml}$ per year in controls). In regional analyses, all patients had a smaller left temporal lobe at baseline (left mean $31.9 \mathrm{ml}$, right mean $49.2 \mathrm{ml}$ ), however the mean rate of atrophy was significantly greater in the right temporal lobe (right $3.9 \mathrm{ml}$ per year, left $2.8 \mathrm{ml}$ per year). Similarly, whereas the left hippocampus was smaller at baseline (left mean $1.92 \mathrm{ml}$, right mean $2.41 \mathrm{ml}$ ) mean atrophy rate was significantly greater in the right hippocampus (right $0.17 \mathrm{ml}$ per year, left $0.10 \mathrm{ml}$ per year). Conclusions: These findings suggest that as SD evolves, atrophy is initially more prominent on the left but then the rate of loss in the right temporal lobe overtakes the left, consistent with the later development of symptoms attributable to right temporal lobe dysfunction in SD. Our findings show that MRI measures of temporal lobe volume loss would provide a feasible and sensitive index of disease progression in SD: whereas whole-brain and hippocampal volume measures have been advanced as candidate biomarkers in other degenerative dementias, in SD temporal lobe volumetry would require fewer patients to detect the same level of effect. 